\title{
Internal force calculation of long span suspension bridge under vertical load based on elastic foundation beam algorithm
}

\author{
Ma Yurong ${ }^{1,2}$, Han Qianwen ${ }^{1 *}$, Wang Feng ${ }^{1,2}$, Li Haixia $^{1}$ \\ ${ }^{1}$ School of Urban Construction, Anhui Xinhua University, Hefei 230088, China \\ ${ }^{2}$ Key Laboratory of Building Structure of Anhui Higher Education Institutes, Anhui Xinhua University, Hefei 230088, China
}

\begin{abstract}
The calculation of suspension bridge under vertical load is the most important content of suspension bridge design calculation and the most important basis of main component design. Combined with the knowledge of structural mechanics, this paper puts forward the elastic foundation beam algorithm, and deduces the important formulas for the cross-section design and strength comparison of sling, cable and stiffening beam. In use, as long as the parameters are brought into the formula, it is more convenient and fast compared with the complex software modeling. The method proposed in this paper is used to check the strength of the completed Japanese Guanmen bridge, and the results are accurate enough. It is fast and reliable to use this method in the preliminary design and rapid safety assessment of suspension bridge.
\end{abstract}

\section{Introduction}

Nowadays, the calculations of long span suspension bridge are completed by bridge structure analysis software such as Dr. bridge, Midas and ANSYS. The numerical accuracy calculated by these software is very high, but it is highly professional, and it needs professionals to complete. Moreover, using bridge software calculation, it needs very complex modelling work, and the workload is very large. In the early stage of engineering project planning, the workload of software modelling analysis is relatively too large. In this paper, an elastic foundation beam algorithm is proposed, which only calculates the internal force used in the section design of suspension bridge sling, cable and stiffening beam by hand without the help of bridge software. With this method, the internal force of each sling, each section of cable and stiffening beam of long span suspension bridge can be calculated within 2 working days. Through the calculation of the completed bridge, the data is very accurate. Using this method, the pre evaluation stage calculation of the project can be carried out more quickly, and the software calculation results can be checked, which reduces a lot of complex modeling work for designers.

Huang Wenli et al. Used MIDAS/Civil modelling to put forward a form finding and internal force calculation method for decorative cables of suspension bridges [1]. Based on the design of a super long span steel box girder suspension bridge, Pi Fuyan and others carried out comparative analysis on the main design parameters such as the ratio of vertical span of main cable and the ratio of side to middle span of stiffening beam, established the calculation model of spatial structure of suspension bridge by using the general finite element analysis software, and calculated and analyzed the static performance of suspension bridge under permanent load, Analysis of the influence of loading length different from train load on the force and deformation of suspension bridge structure system under dynamic action [2]. Liu Yi et al studied the calculation method of cable shape and internal force of suspension bridge under dead load. First, the approximate position of main cable is given by using catenary formula. Then, through the analysis process of structure splitting and combination, the calculation steps of cable shape and internal force are given [3].

In this paper, the mechanical model of suspension bridge is established, and the calculation formula of internal force of sling, cable and stiffening beam is derived, which is verified by an example. At present, this kind of method is rarely mentioned in the literature.

\section{Calculation principle and formula}

The structural diagram of suspension bridge is shown in Figure 1.

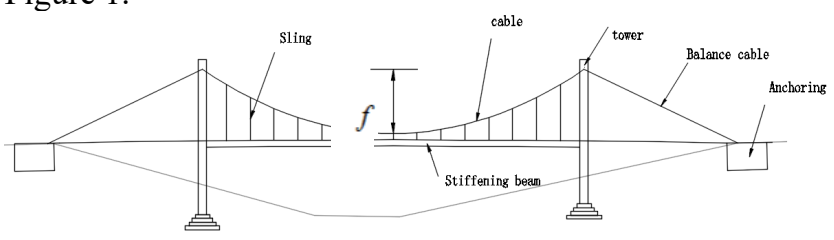

Fig. 1. structural diagram of suspension bridge.

The calculation structure of this paper is shown in Figure 2.

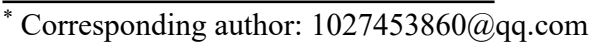




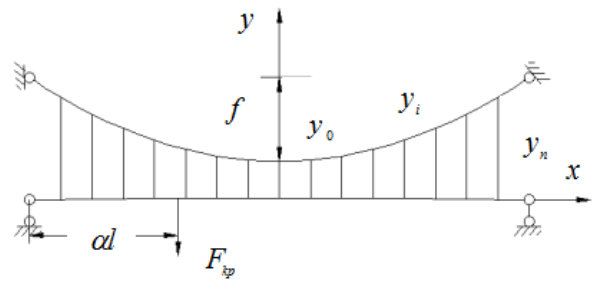

Fig. 2. Calculation structure diagram

There is one cable on each side along the length direction of the bridge, and the vertical sling is connected under the cable. There are $(2 n-1)$ slings $(2(2 n-1)$ in total) on each side. Each cable is divided into $2 n$ sections, the horizontal distance of each section is $d$, and the length of each section is,

$$
\begin{gathered}
\Delta l_{i}=\sqrt{d^{2}+\Delta y_{i}^{2}} \\
\Delta y_{1}=y_{1}-y_{0}, \ldots \Delta y_{n}=y_{n}-y_{n-1}
\end{gathered}
$$

Where, $y_{n}$ is the distance from the cable end to the bridge deck,

$$
y_{n}=y_{0}+f
$$

The cable equation is as follows,

$$
y=\frac{x^{2}}{A}+y_{0}
$$

When the cable axis is a parabola and the distance between slings is $d, y^{\prime \prime}$ is a constant, then the quadratic difference equation $\partial \Delta y$ of $y$ is also a constant.

$$
\begin{gathered}
y^{\prime \prime}=\frac{d^{2} y}{d x^{2}} \\
\partial \Delta y=\Delta y_{i+1}-y_{i}
\end{gathered}
$$

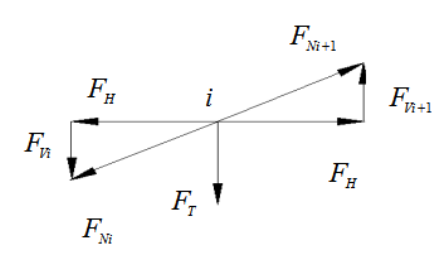

Fig. 3. Force diagram at node.

It can be seen from Fig. 3 that,

$$
\sum Y=0, F_{T i}=F_{V i+1}-F_{V i}=\frac{\partial \Delta y}{d} F_{H}
$$

$\partial \Delta y$ is a constant, then $F_{T i}$ is also a constant. Therefore, in the structure shown in Fig. 2, under the action of $F_{k p}, q_{k}$ (there is no external load on the cable and sling), the tension generated by each sling is the same. The system shown in Figure 2 is One Degree of indeterminate when the force method is used. The basic system of force method is shown in Figure 4.

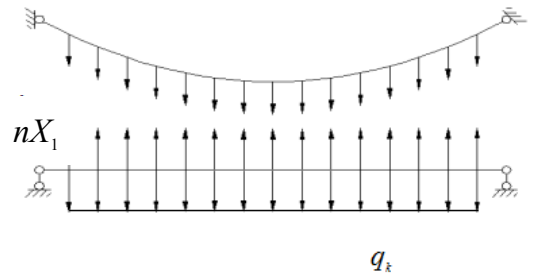

Fig. 4. Basic system of force method

The equation of force method is as follows,

$$
\delta_{11} X_{1}+\Delta_{1 P}=0
$$

(1) The coefficient $\delta_{11}$ consists of three parts

$\delta_{11}^{0}$ is the vertical displacement of the beam at $C$ points in the middle span of the stiffening beam under the action of $(2 n-1) \quad X_{1}=1$ concentrated forces.

$\delta_{11}^{T}$ is the displacement of $C$ points in the middle of the beam span caused by $(2 n-1)$ slings under the action of tension $F_{T}=X_{1}=1$.

$\delta_{11}^{N}$ is the displacement at $C$ in the middle of the beam span under the action of $\bar{F}_{N i}, \bar{F}_{N i}$ is the internal force of each section of cable ( $2 n$ sections ) under the action of $X_{1}=1$.

$$
\delta_{11}=\delta_{11}^{0}+\delta_{11}^{T}+\delta_{11}^{N}
$$

There are $(2 n-1) \quad X_{1}=1$ concentrated forces on the stiffening beam, which are replaced by $\bar{q}=\frac{1}{d}$ ( $d$ is sling spacing). After calculating the support reaction, the $\bar{M}_{1}$ equation of the beam is listed as follows,

$$
\begin{gathered}
\bar{M}_{1}=\frac{1}{2} \frac{1}{d} l x-\frac{1}{2} \frac{1}{d} x^{2} \\
\delta_{11}^{0}=\frac{1}{E I} \int_{\phi}^{l} \bar{M}_{1}^{2} d x=\frac{l^{5}}{120 \times d^{2} E I} \\
\delta_{11}^{T}=\frac{1}{E_{S} A_{S 2}} \sum y
\end{gathered}
$$

Where $y$ is the length of each sling.

$$
\begin{gathered}
\delta_{11}^{N}=\frac{1}{E_{S} A_{S 1}} \sum \bar{F}_{N}^{2} \Delta l=\frac{1}{E_{S} A_{S 1}}\left(\frac{1}{\partial \Delta y}\right)^{2} \sum \Delta l^{3} \\
\Delta l=\sqrt{d^{2}+\Delta y_{i}^{2}}
\end{gathered}
$$

Where $\Delta l$ is the length of each cable, $E_{S}$ is the elastic modulus of the cable and sling, $A_{S 2}$ is the cross-sectional area of the sling, $A_{S 1}$ is the cross-sectional area of the cable.

(2) Constant $\Delta_{1 p}$

As the dead weight of cable and sling has little effect on $\Delta_{1 p}$, it is not included in the calculation of $\Delta_{1 p} . \Delta_{1 p}$ 
is caused by the load acting on the beam and the dead weight of the beam.

(1) $\Delta_{1 p}^{q}$ under dead load $q_{k}\left(q_{k}=q_{k p}+\right.$ dead weight of half side beam)

$$
\Delta_{1 p}^{q}=\frac{1}{E I} \int \bar{M}_{1} M_{P}^{q} d x=\frac{-l^{5} q_{k}}{120 d E I}
$$

(2) $\Delta_{1 p}^{p}$ under the action of live load $F_{k p}$

$$
\Delta_{1 p}^{p}=\frac{1}{E I} \int \bar{M}_{1} M_{p}^{p} d x=\frac{-l^{4}}{24 d E I}\left(\alpha-2 \alpha^{3}+\alpha^{4}\right) F_{k p}
$$

Where $\alpha l$ is the action position of $F_{k p}$ on the beam.

$(15)+(16)$ is as follows,

$$
\Delta_{1 p}=\Delta_{1 P}^{q}+\Delta_{1 p}^{p}=\frac{-l^{4}}{d E I}\left[\frac{q_{k} l}{120}+\left(\alpha-2 \alpha^{3}+\alpha^{4}\right) \frac{F_{k p}}{24}\right]
$$

According to the formula (8), it can be concluded that,

$$
X_{1}=-\frac{\Delta_{1 P}}{\delta_{11}}
$$

According to the result of formula (18), it can be concluded that,

(1) Sling tension

$$
F_{T}=X_{1}
$$

(2) Cable

Horizontal component,

$$
F_{H}=\frac{d}{\partial \Delta y} X_{1}
$$

The tension of section $i$,

$$
F_{N i}=\frac{\Delta l_{i}}{d} F_{H}=\frac{\Delta l_{i}}{\partial \Delta y} X_{1}
$$

Cable end tension,

$$
F_{N}=F_{N \max }=\frac{\Delta l_{\max }}{\partial \Delta y} X_{1}
$$

(3) If $X_{1}$ is changed into uniform load $\left(\frac{X_{1}}{d}\right)$, and there is $q_{k}$ original uniform load on the beam, then $q_{1}=\frac{X_{1}}{d}-q_{k}$ is the distributed load acting on the stiffening beam (upward).Under the action of $q_{1}$ and $F_{k p}$, the load diagram on the beam is shown in Figure $5 a$, and the drawing of stiffening beam $M$ diagram is shown in Figure $5 b$.

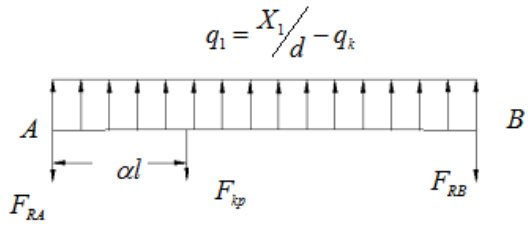

(a) Force diagram of stiffening beam

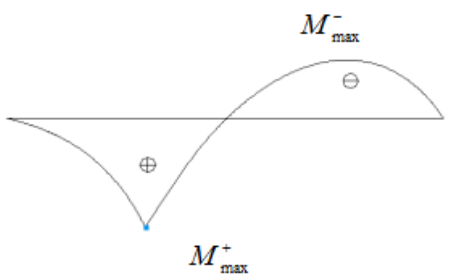

(b) M diagram of stiffening beam

Fig. 5. Force diagram and $M$ diagram of stiffening beam In the fig.5,

$$
\begin{gathered}
M_{\text {max }}^{+}=\frac{l}{2} \alpha(1-\alpha)\left(2 F_{k p}-q l\right) \\
M_{\text {max }}^{-}=\frac{F_{R B}^{2}}{2 q_{1}}
\end{gathered}
$$

$$
\text { Location } x_{0}=\frac{F_{R B}}{q_{1}}
$$

(4) $\alpha l$ take different values, calculate the corresponding $M_{\max }^{+}$, draw the envelope diagram of $M_{\max }^{+}$, as shown in Figure 6 . When the external force on the stiffening beam is only $q_{k p}=10.5 \mathrm{kN} / \mathrm{m}, F_{k p}=360 \mathrm{kN}$ (or $q_{k p}=7.875 \mathrm{kN} / \mathrm{m}, F_{k p}=270 \mathrm{kN}$ ), $M_{\max }^{\text {absolute }}$ is located near $l / 5$ or $4 l / 5$.

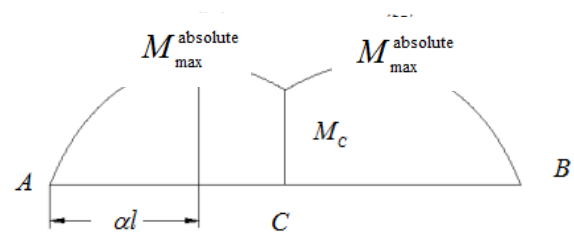

Fig. 6. Envelope diagram of $M_{\max }^{+}$

When the dead weight of the stiffening beam is added to the dead load $q_{k}$, the $M_{\max }^{\text {absolute }}$ position is $\alpha l=\frac{l}{4} \sim 0.3 l$, and in most cases, it is $\frac{l}{4}$ (or $\frac{3 l}{4}$ ), and the formula (23) is as follows,

$$
M_{\max }^{+}=\frac{6 l}{32}\left(2 F_{k p}-q_{1} l\right)
$$

The corresponding formula (12) is as follows,

$$
\Delta_{1 p}=\frac{-l^{4}}{d E I}\left(\frac{q_{k} l}{120}+\frac{19}{2048} F_{k p}\right)
$$

When $F_{k p}$ placed at position $0.3 l$, the formula (23) is as follows, 


$$
M_{\max }^{+}=0.21 l\left(2 F_{k p}-q_{1} l\right)
$$

The corresponding formula (12) is as follows,

$$
\Delta_{1 p}=\frac{-l^{4}}{d E I}\left(\frac{q_{k} l}{120}+\frac{2541}{240000} F_{k p}\right)
$$

Through the above calculation, the section design and strength check of the stiffening beam are based on the size of $M_{\max }^{+}$.

(5) The section design and strength check of sling and cable is to place $F_{k p}$ in $C(\alpha=1 / 2)$. In this case, the formula (12) is as follows,

$$
\Delta_{1 p}=\frac{-l^{4}}{d E I}\left(\frac{q_{k} l}{120}+\frac{5}{384} F_{k p}\right)
$$

$X_{1}=-\frac{\Delta_{1 P}}{\delta_{11}}$, so the tension of the longest sling is,

$$
F_{T_{\text {max }}}=X_{1}+G \times 9.81
$$

Here $\mathrm{G}$ is the weight of the sling itself.

The maximum tension $F_{N \max }$ of the cable occurs at the end of the cable, near the the tower, the length of this cable is $\Delta l_{\max }=\sqrt{d^{2}+\Delta y_{\max }^{2}}$. The parts produced by $F_{T \text { max }}$ are as follows,

$$
F_{N \text { max }}^{\prime}=\frac{\Delta l_{\text {max }}}{\partial \Delta y} F_{T \text { max }}
$$

The self weight of the cable is produced by the following parts,

$$
\begin{array}{r}
F_{N \max }^{\prime \prime}=\frac{1}{8} q \times \frac{l^{2}}{f} \times \frac{\Delta l_{\max }}{d} \\
q=\frac{G}{l}=\frac{\pi}{4} D_{N}^{2} \times 7.85 \times 9.81 \times \frac{S}{l}
\end{array}
$$

Where $\mathrm{q}$ is the uniformly distributed load due to the dead weight of the cable, $G$ is the total weight of the cable.

$$
F_{N \max }=F_{N \max }^{\prime}+F_{N \text { max }}^{\prime \prime}
$$

$F_{T \text { max }}$ and $F_{N \text { max }}$ here are the basis for the section design and strength check of sling and cable.

\section{Examples}

Japan's Guanmen bridge[4], as shown in Figure 7 (this example is characterized by a suspension bridge with side spans, including wind load and seismic load).

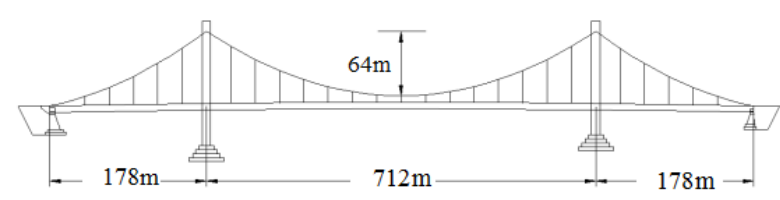

Fig. 7. Schematic diagram of Guanmen bridge in Japan

The bridge was built in 1973, with a total span of $1068 \mathrm{M}(178+712+178)$. It is a three span steel truss suspension bridge. The main cable span is $712 \mathrm{M}$, the side span is $178 \mathrm{M}$, the tower height is $133.8 \mathrm{M}$, the stiffening beam is $702 \mathrm{M}$, the side span is $167.2 \mathrm{M}$, the beam height is $9 \mathrm{M}$, the width is $28.5 \mathrm{M}, I_{z}=2.916 \mathrm{~m}^{4}$, the main cable sag is $64 \mathrm{M}$, the side span cable sag is $3.909 \mathrm{M}$, the main cable $A_{S 1}=0.599 \mathrm{~m}^{2} \quad(2 \times 154 \times 91 \phi 5.04 \mathrm{~mm})$, $E=2 \times 10^{5} \mathrm{Mpa}$, the distance between the two trusses is $29 \mathrm{M}$, and the truss section is $10.35 \mathrm{M}$ long, When the main girder is designed, the dead load is $120.86 \mathrm{kN} / \mathrm{m}$, the wind load is $18.85 \mathrm{kN} / \mathrm{m}$, and the seismic load is $4.57 \mathrm{kN} / \mathrm{m}$. When the main cable is designed, the dead load is $25.66 \mathrm{kN} / \mathrm{m}$, the wind load is $3.02 \mathrm{kN} / \mathrm{m}$, and the seismic load is $1.23 \mathrm{kN} / \mathrm{m}$.

For the suspension bridges built on the coastal rivers and harbors, the safety factor is too large in design because of typhoon. The safety factor of the sling is $K>4$, cable is $K>2.5$ and stiffening beam is $K>3$ (because the diameter of the sling is much smaller than that of the cable, the sling is prone to flutter under the action of wind and rain, so the safety factor is greater than that of the cable).Because Japan is located in the sea and earthquake prone area, the design of suspension bridge should consider not only the self weight but also the wind load and earthquake load.Wind load and earthquake load are considered in the design of Japan's Guanmen bridge.

This example is of great reference value for the design of suspension bridges in earthquake areas.Based on the above data, the elastic foundation beam algorithm proposed by the author is used for calculation. The calculation is as follows,

Take the distance between the slings as $d=8.9 \mathrm{~m}, 79$ slings under each cable, and the cable is made of $\phi 60$ high strength steel cable. The tensile force of each sling is $4524 \mathrm{kN}\left(A_{s 2} \times 1600 \mathrm{Mpa}\right)$, and $E_{s} A_{s 2}=E I / 500$ is used for calculation ; one cable can bear the tensile force of $447200 \mathrm{kN}\left(A_{S 1} \times 1600 \mathrm{Mpa}\right)$, and $E_{s} A_{s 1}=E I / 5.2$ is used for calculation, the truss beam is welded with 16 manganese steel, and each truss can bear the bending moment of $220320 \mathrm{kN} \cdot \mathrm{m}$. The cable is $l=712 \mathrm{M}$, $f=64 \mathrm{~m}$, then $f / l=1 / 11.125$ and the cable equation is $y=\frac{4 x^{2}}{7921}+4$.From this equation, it can be calculated that,

$$
\begin{aligned}
& \partial \Delta y=0.08 \\
& y_{\text {max }}=64.84 \mathrm{~m}(x= \pm 39 d)
\end{aligned}
$$




$$
\begin{aligned}
& \Delta l_{\text {max }}=9.444 \mathrm{~m} \\
& \frac{\Delta l_{\text {max }}}{d}=1.0612 \\
& \sum y=y_{0}+\sum y_{i}=1918 \mathrm{~m} \\
& \sum \Delta l^{3}=61890 \mathrm{~m}
\end{aligned}
$$

Coefficient,

$$
\begin{gathered}
\delta_{11}^{0}=\frac{702^{5}}{120 \times 8.9^{2}} / E I=1.793598 \times 10^{10} / E I \\
\delta_{11}^{T}=\frac{500}{E I} \times 1918=9.59 \times 10^{5} / E I \\
\delta_{11}^{N}=\frac{5.2}{E I}\left(\frac{1}{0.8}\right)^{2} \sum \Delta l^{3}=5.028563 \times 10^{7} / E I \\
\delta_{11}=1.798719 \times 10^{10} / E I
\end{gathered}
$$

Constant,

$\Delta_{1 p}$ in the calculation formula (because the $\Delta_{1 P}$ caused by the dead weight of cable, wind load and earthquake load is very small, it is not included in $\Delta_{1 P}$ )

$q_{k}=(120.86+18.85+4.57) / 2+10.5=82.72 \mathrm{kN} / \mathrm{m}$

When $F_{k p}=360 \mathrm{kN}$ is placed in the middle of the span, check the cable strength,

$$
\begin{gathered}
\Delta_{1 P}=\frac{-702^{4}}{8.9 E I}\left(\frac{82.73}{120} \times 702+\frac{5}{384} \times 360\right) \\
=\frac{-1333.409 \times 10^{10}}{E I} \\
X_{1}=-\frac{1333.409}{1.79872}=741.31 \mathrm{kN}
\end{gathered}
$$

(1) Sling

$$
F_{T \text { max }}=X_{1}+G=741.31+14.04=755.35 \mathrm{kN}
$$

Here $\mathrm{G}$ is the weight of the longest sling.

Safety factor $K=\frac{4524}{755.35}=5.99$

(2) Cable

$$
\begin{aligned}
& F_{N \text { max }}^{\prime}=\frac{\Delta l_{\text {max }}}{\partial \Delta y} F_{T_{\text {max }}}=\frac{9.444}{0.08} \times 755.35=89169 \mathrm{kN} \\
& q^{0}=q_{1}+q_{2}+q_{3}=25.66+3.02+1.23=29.91 \mathrm{kN}
\end{aligned}
$$

Here $q_{1}$ is the dead weight of the cable, $q_{2}$ is the wind load acting on the cable, $q_{3}$ is the seismic load acting on the cable.

$$
\begin{aligned}
& F_{N \text { max }}^{\prime \prime}=\frac{1}{8} q^{0} \frac{l^{2}}{f} \times \frac{\Delta l_{\text {max }}}{d} \\
& =\frac{1}{8} \times 29.91 \times \frac{712^{2}}{64} \times 1.0612=31427 \mathrm{kN}
\end{aligned}
$$

Here $l$ is the horizontal span of cable.

$$
\begin{aligned}
& F_{N \text { max }}=F_{N \text { max }}^{\prime}+F_{N \text { max }}^{\prime \prime}=120596 \mathrm{kN} \\
& \text { (horizontal pull } F_{H}=113641 \mathrm{kN} \text { ) }
\end{aligned}
$$

Safety factor: $K=\frac{44700}{120596}=3.708$

(2) When $F_{k p}=360 \mathrm{kN}$ is placed at $\frac{1}{4} l$ (or $\frac{3}{4} l$ ), check the strength of the stiffening beam:

$$
\begin{aligned}
& \Delta_{1 P}=\frac{-702^{4}}{8.9 E I}\left(\frac{82.73}{120} \times 702+\frac{19}{2408} \times 360\right) \\
& =\frac{-1329.73185 \times 10^{10}}{E I} \\
& X_{1}=\frac{1329.73185}{1.79872}=793.266 \\
& q_{1}=\frac{X_{1}}{8.9}-82.73=0.3336 \mathrm{kN} / \mathrm{m}(\uparrow) \\
& M_{\max }^{+}=2 \times \frac{3 \times 702}{32}(2 \times 360-702 \times 0.3336) \\
& =63945.2 \mathrm{kN} / \mathrm{m} \\
& \text { Safety factor } K=\frac{220320}{63945.2}=3.445 .
\end{aligned}
$$

\section{Conclusions}

According to the elastic foundation beam algorithm proposed in this paper, the strength of the completed Japanese Guanmen bridge is checked, which has enough accuracy. This paper deduces the important formulas for the section design and strength comparison of sling, cable and stiffening beam. In use, only the parameters are brought into the formula. Compared with the complex software modeling, it is more convenient and fast. This algorithm can be used for the preliminary design of suspension bridge, and can also check the calculation results of bridge software and rapid safety assessment.The following aspects need to be further studied.

(1) The algorithm can be programmed into a computer program, and the calculation results can be obtained instantly by inputting the design parameters of the suspension bridge.

(2) The algorithm is extended to the calculation and design of cable-stayed bridge and arch bridge.

At present, there are few literatures about this kind of algorithm, and the research is not mature enough. This method needs to be used in practical engineering, and its practicability can be proved by more engineering data, so that more people will know this kind of algorithm and further optimize it. There is still a long way to go for the development of beam algorithm on elastic foundation.

\section{Acknowledgements}

This paper is one of the phased achievements of the key project of Horizontal Topic "Experimental study on the basic mechanical properties of reinforced fiber reinforced concrete with high performance ductility" (2018cxy006),Natural Science Research in Universities of Anhui Province "Study on dynamic mechanical properties of heterogeneous materials based on extended finite element method" (KJ2019A0882),Anhui Province 
Quality Engineering Project "Teaching team of civil engineering" (2019jxtd118).

\section{References}

1. Huang W, Zheng M.W, Xu F, Zhou J.J. (2015) Form finding and internal force calculation of decorative cable of landscape suspension bridge. Journal of Northeast University, 36:143-147.

2. Pi F.Y, Lei J.Q, Lu W.L, Chen J.C. (2021) Preliminary study on Design of super long span steel box girder suspension bridge for highway and railway use. Railway Engineering, 61:1-4.

3. Liu Y, Li A.Q, Guo T. (2007) Calculation method of cable shape and internal force of suspension bridge under dead load. Bridge Construction, 03:24-26.

4. Bridge Engineering Bureau of Ministry of Railways, Institute of bridge science.(1996) suspensionbridge. Science and Technology Literature Press, Beijing. 